NASA Technical Memorandum 105908

AIAA-92-4713

\title{
Concurrent Optimization of Airframe and Engine Design Parameters
}

Thomas M. Lavelle, Robert M. Plencner, and Jonathan A. Seidel Lewis Research Center Cleveland, Ohio

Prepared for the

Fourth Symposium on Multidisciplinary Analysis and Optimization cosponsored by the AIAA, USAF, NASA, and OAI

Cleveland, Ohio, September 21-23, 1992

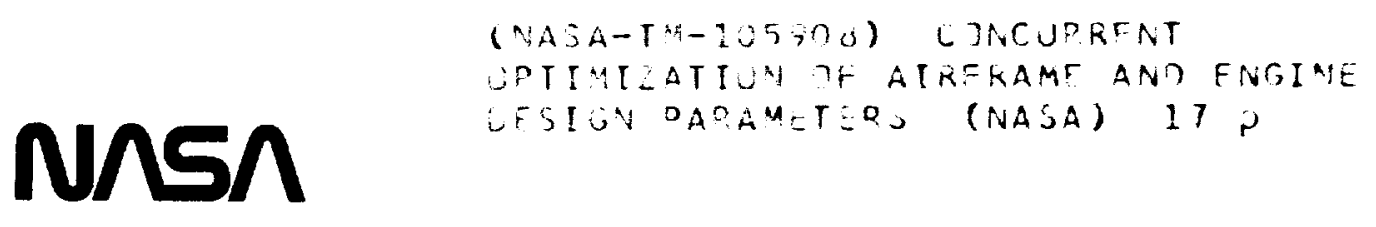

$193-1240 ?$

Unclas 


\title{
CONCURRENT OPTIMIZATION \\ OF AIRFRAME AND ENGINE DESIGN PARAMETERS
}

\author{
Thomas M. Lavelle \\ Robert M. Plencner \\ Jonathan A. Seidel \\ NASA Lewis Research Center, Cleveland, Ohio 44135
}

\begin{abstract}
An integrated system for the multidisciplinary analysis and optimization of airframe and propulsion design parameters is being developed. This system is known as IPAS, the Integrated Propulsion/Airframe Analysis System. The traditional method of analysis is one in which the propulsion system analysis is loosely coupled to the overall mission performance analysis. This results in a time consuming iterative process. First, the engine is designed and analyzed. Then, the results from this analysis are used in a mission analysis to determine the overall aircraft performance. The results from the mission analysis are used as a guide as the engine is redesigned and the entire process repeated. In IPAS, the propulsion system, airframe, and mission are closely coupled. The propulsion system analysis code is directly integrated into the mission analysis code. This allows the propulsion design parameters to be optimized along with the airframe and mission design parameters, significantly reducing the time required to obtain an optimized solution.
\end{abstract}

\section{Introduction}

The purpose of performing a mission analysis within the Aeropropulsion Analysis Office (AAO) at the NASA Lewis Research Center is to assess the benefits of advanced propulsion concepts and technologies for future airbreathing aerospace vehicles. This assessment is based on the performance of the vehicle over a particular mission. This process is depicted in Figure 1. For each system being studied it is necessary to determine the combination of aircraft and engine design variables that will yield the optimum solution. It is necessary to determine the best match for this system in order to adequately compare it to other possible solutions.

The overall performance of the aircraft and propulsion system is determined from computer simulations which combine the characteristics of the engine, airframe, and mission as shown in Figure 2.

The traditional method is one in which the propulsion analysis and mission analysis are handled separately. The first step is to analyze the propulsion system. This is done by creating a computer simulation of the engine. The computer simulation is then used to generate a table of performance data. This data table contains fuel consumption as a function of Mach Number, altitude, and thrust level. A weight assessment of the total propulsion system is also conducted. This information will then be used to perform a mission analysis.

A mission analysis combines engine data from the propulsion analysis with aircraft aerodynamic and weight data to determine overall performance results such as takeoff gross weight (TOGW) and takeoff field length (TOFL). The mission analysis code does allow for variations in the aircraft design variables of engine size and wing size. Thus, it is possible to determine the optimum aircraft solution for a fixed engine design. This can either be done by using an optimizer or by the graphical thumbprint method. A typical thumbprint is shown in Figure 3. The thumbprint shows aircraft gross weight as a function of engine size and wing size. The optimum solution is the lightest aircraft that satisfies the constraints. This solution is the design indicated by the solid circle. The arrows indicate the path taken 
by an optimizer from the initial guess (represented by an open circle). However, the mission code does not allow for variations in engine design parameters. The engine design is fixed at the time the engine data is produced. In order to determine the optimum combination of aircraft and engine design it is necessary to try many different engine designs. For each engine design the optimum aircraft solution is determined. These optimum solutions are then compared to determine the best overall design choice. This method can be very time consuming. It is shown in Figure 4.

This process has been improved by directly coupling the propulsion analysis codes and mission analysis codes with an optimizer. The engineer defines the baseline aircraft and cycle. The optimizer acts as the main program, running the analysis and changing the design parameters to arrive at a solution. This method, called IPAS, is shown in Figure 5. IPAS would greatly reduce the time required to reach an optimum solution by performing all the design iterations necessary. The engineer would be free to spend more time analyzing results and developing new configurations.

This paper will describe the analysis codes used in IPAS and the methods used to link them. This paper will also give an example of IPAS being applied to a supersonic transport mission. The goal of the analysis is to determine the minimum TOGW aircraft subject to the constraints of noise and TOFL.

\section{Integration of Analysis Codes}

Five analysis codes are directly coupled in IPAS. The cycle analysis code used is NEPP, the NASA Engine Performance Program (ref. 1). The mission analysis code used is FLOPS, the Flight OPtimization System (ref. 2). The engine weights are computed using WATE, Weight Analysis of Turbine Engines (ref. 3). The inlet and nozzle performance are calculated using INSTAL (ref. 4). The noise analysis is calculated using FOOTPR (ref. 5).

\section{Cycle Analysis}

NEPP is an engine simulation code that will perform one dimensional steady state thermodynamic analysis of turbine engine cycles. Engine performance is calculated using component performance maps. The engine is defined by describing the flowpath that connects the components and the controls necessary to balance the engine. These controls tell the code how to balance the engine by listing what parameters can be varied and what values have to be matched. Once the engine and control scheme have been defined it is possible to generate performance data.

In order run NEPP as a subroutine, it is necessary to have the control scheme fixed before executing IPAS. This can prove to be difficult. Often, when running NEPP stand alone it is necessary to modify the input in some way to get an answer. This may be done by tuming an additional control on or changing the spacing between points. An example of this would be increasing the number of points in the throttle curve so NEPP can take smaller steps in thrust level. This option is not available when running NEPP as a subroutine. The control scheme must be able to handle any situation that may occur. If it is not able to then it must be modified. There may be cases when the cycle proves to be 100 complex to run concurrently. A good check is to run an engine envelope similar to what the mission code would require. If it is possible to get all the engine data by only varying altitude, Mach Number, and power setting, then it is a good sign that the control scheme is adequate. If the NEPP simulation does not give consistent results then the control scheme must be modified.

Once NEPP had been modified to work as a subroutine, the next step was to determine how to exchange propulsion data between it and the mission code. The first method that was tried was a direct method. Every time the mission analysis needed propulsion data, the cycle analysis code was executed. This proved to be a very time consuming way of doing things due to the large number of mission propulsion calls. One method employed to try to speed up this process was to create a running library of engine data. Tolerance levels were input by the user. If the propulsion input values of altitude, Mach number, and thrust, were all within the tolerance level of a previous call the output data from the previous point were used. If the input values were not within tolerance level, NEPP would be executed and the data from this case would be added to the library. This method was successful in greatly shortening the execution time without having a large effect on the accuracy. 
Another approach to exchanging data between NEPP and FLOPS was the use of an automatic table generator. A table generator had already been developed and incorporated into the FLOPS code using the cycle analysis code QNEP (ref. 6). The QNEP routines were removed and replaced with NEPP routines. Before the mission was run, NEPP would generate a full envelope of propulsion data including any propulsion data needed for such things as noise calculations. This method had several advantages over the previous one. NEPP uses the previous converged case as a starting point for the next case. When using a table generator the spacing and order of the engine data points is controlled. Since the engine data requests are in order, this yields small steps between engine points. This makes NEPP run much smoother and more reliably. It is also a more organized procedure since all the engine related data is generated at one time. This is the method that was used for all of the examples presented in this paper.

\section{Installation}

The next step in integrating the codes was to define the inlet operation. In order to use the INSTAL code it is necessary to define a performance map and a capture area for the inlet. The performance map is read from a database. The capture area was determined by the quantity of airflow the engine needed at cruise. Assuming no spillage, the inlet should supply just enough air for the engine. The capture area was determined by running a sample cruise point and sizing the inlet to provide the airflow required.

\section{Noise}

The next step in integrating the analysis codes was to include the noise computation. FOOTPR had already been integrated into FLOPS. The only thing that had to be done was to automatically generate the propulsion related FOOTPR inputs. For this supersonic transport case it was assumed that the jet was the dominant noise source. The engine related noise data was determined by generating a throttle curve at an altitude of 500 feet and a Mach Number of .30. This point is assumed to be representative of the entire takeoff. The throttle curve has jet velocity, area, and temperature, as a function of power setting. This data is combined with a takeoff trajectory generated by FLOPS and input into FOOTPR to determine the takeoff noise.

\section{Engine Weight}

The final step in linking the analysis codes was to find a way to determine the engine weight and size as a function of the design variables involved. The engine weight and geometric flowpath are calculated using the WATE code. This flowpath is deternined by combining NEPP output, cycle conditions, with WATE aero-mechanical inputs, Mach Numbers, turbine loadings, etc. The user must determine if this flowpath is acceptable. If it is not then the WATE inputs must be modified and the case renun. Figure 6 shows an acceptable flowpath. This was generated by adjusting the WATE inputs until the components lined up. The pressure ratio of this engine was then increased without changing the WATE inputs. This resulted in Figure 7. Obviously the turbine is too far out. What would be required is to add a turbine stage or increase the turbine loading to bring the turbine in. It would be up to the user to modify these inputs until an acceptable flowpath is obtained. This make WATE an interactive code by nature. Several methods were tried to make WATE non interactive in IPAS.

The first method that was tried was to have IPAS automatically adjust the WATE inputs until an acceptable flowpath was obtained. Simple rules were written that told IPAS what an acceptable flowpath was and what inputs to change if it was not acceptable. This method was partially successful. IPAS was able to generate engines with reasonable geometries. However, this method did not work well with the optimizer. A small change in the design overall pressure ratio could result in a compressor or turbine stage being added or dropped. This will cause a step change in the engine weight. This is shown in Figure 8 . The optimization techniques currently being used were unable to cope with this sudden change. In addition, these rules are specific to the engine being used in this study. A new system of rules would have to be produced for every new cycle studied.

The next method that was tried was to map the engine weight as a function of the design parameters involved. Generating this type of map is a time consuming process. This is not a desirable way for determining engine weights in IPAS. The most efficient way of determining the weight of this engine was found by plotting the engine weight as a function of the design airflow. Figure 9 shows engine weight plotted as a function of the design 
airflow over a wide range of values of the design parameters involved. This indicates that for the engine used in this study it is possible to schedule the weight as a function of the design airflow only. This is the method that was used in this paper. This trend is specific to the engine being studied in this paper. It may not hold for different cycles with different design parameters. A more general method for determining engine weight should be developed for the IPAS system.

The nozzle and inlet weights were calculated using the data in Figures 10 and 11 . These figures represent semi-analytical algorithms generated at NASA-Lewis. The inlet weight was a direct function of the capture area. The capture area was determined when the inlet is sized by the INSTAL code. The nozzle weight was a function of the jet velocity and airflow at takeoff. The airflow and jet velocity were calculated using NEPP.

\section{Optimizer}

For these example cases the FLOPS intemal optimizer was used. The FLOPS optimizer uses the Sequence of Unconstrained Minimizations Technique with a Fiacco-McCormmick penalty function with quadratic extension.

This optimizer proved to be very difficult to use. For many of the cases presented in this paper it took several nuns before what were believed to be optimum results were achieved. There were several warning signs that were looked for to determine whether or not the solutions obtained were the true optimums. The first sign that a solution was bad was if the constraints were not met at all. The second warning sign was if a constraint was met by a large margin. For example, if the takeoff field length was well below the allowable limit it meant that the airplane could possibly use a smaller, lighter engine then the optimizer indicated. Common sense was applied to the results to see if they made sense or not. If they did not the optimizer set up would be modified and the case rerun. This was done by varying the starting point or changing other optimizer inputs. There was no scientific method to this. The inputs were changed until the results were reasonable. This brought out another major weakness in the IPAS system. Currently the optimization schemes being used are unreliable. If IPAS is to be used to its fullest potential then the optimizer must be able to find the true solution with a minimal amount of work.

\section{TBE Engine}

The engine chosen for this study was an advancedtechnology turbine bypass engine (TBE) (ref. 7). This type of engine has been identified as one of the promising candidate cycles for a next-generation supersonic transport. The basic engine cycle is similar to that of a turbojet operating with fixed area choked turbines. The advantage of the TBE over the turbojet is a bypass valve which allows the cycle to maintain constant turbine corrected airflow throughout the flight envelope without throttling. By bypassing a minimal amount of compressor discharge air around the bumer and turbines, higher cycle pressures and temperatures can be achieved which yield greater specific thrust. In addition, varying the bypass flow for cruise power adjustments helps balance turbine horsepower and airflow requirements thereby allowing lower bumer temperatures for more efficient cruise. The net effect of the turbine bypass system is high specific thrust at sustained airflows resulting in less spillage and boattail drag. A schematic of the TBE is presented in Figure 12.

A NEPP cycle simulation of the TBE has been developed for use in the HSR, High Speed Research program at NASA Lewis. This simulation was used for this study. There were, however, several modifications that had to be made to prepare the TBE for use in IPAS.

The first modification was to adjust the control scheme to run automatically. This was accomplished successfully with a minimal amount of trouble. The next modification was to map certain engine parameters as a function of the design variables. The design compressor efficiency was scheduled as function of the design compressor pressure ratio. This can be seen in Figure 13. The amount of cooling flow required was scheduled as a function of the maximum turbine inlet temperature. This can be seen in Figure 14. Thus, when the optimizer changes a design variable the affect of these changes on the individual components is automatically taken into account.

\section{Description of Sample Problem}


The test problem for IPAS was a Mach 2.4 high speed civil transport with a 6500 nautical mile mission. The aircraft was equipped with four turbine bypass engines. The aircraft was constrained to a takeoff field length of 11000 feet and FAR stage III noise requirements.

The aircraft design variables were sea level static thrust per engine, wing area, and takeoff scaling factor (TSF). The TSF represents the amount the engine is oversized at takeoff. For example 1.25 indicates that the engine is 25 per cent oversized at takeoff. This means that $1 / 1.25$ or 80 per cent of the full power thrust is used during takeoff. This is done to reduce the jet velocity. The engine design variables were maximum turbine inlet temperature (T4 Maximum) and the engine overall pressure ratio (OPR).

The solution to this problem was the design that yielded the lightest aircraft and also satisfied the constraints. The desired objective function drove the design variables one direction while the constraints may have driven the design variables another direction.

The desire to have a light weight aircraft drove the design process. The engine and wing should generally be as small as possible to minimize the weight of these components. The maximum turbine inlet temperature should be as high as possible to maximize specific thrust. The overall pressure ratio should be as high as allowable to maximize cycle efficiency.

The constraints also drove the design variables. The takeoff field length is determined by the engine size, wing size, takeoff scaling factor, and overall gross weight. Any increase in engine size, wing size, or a decrease in takeoff scaling factor will have a strong tendency to decrease the takeoff field length. The takeoff noise is driven by the maximum turbine inlet temperature and the takeoff scaling factor. A decrease in the maximum turbine inlet temperature or an increase in the takeoff scaling factor will decrease the jet velocity. Since jet velocity is the driving force in noise, this will result in lower noise levels.

Noise is also controlled through nozzle suppression. Currently this is an active area of research in the HSR program. Noise reduction can be achieved by the use of a mixer/ejector nozzle, shown in Figure 12, which will entrain large amounts of ambient air and mix it with high velocity air from the engine during takeoff. This results in lower jet velocities and lower noise levels. Nozzle suppression is the amount of noise reduction this process will yield when compared to ideally expanded conical jet of the primary stream. The overall noise levels are calculated by taking the FOOTPR results, which assumes a conical jet, and subtracting the amount of assumed nozzle suppression. At the present time it is assumed that an advanced ejector nozzle will yield between $10 \mathrm{~dB}$ and $20 \mathrm{~dB}$ of nozzle suppression.

\section{Results}

Several example problems were run to test the viability of IPAS. As stated before, the basic problem was to devise a solution for a Mach 2.4 6500 nautical mile supersonic cruise mission with four TBE engines. The problem was to find the minimum TOGW aircraft subject to the constraints of FAR stage III noise requirements and a TOFL of 11000 feet.

\section{Eirst Example Problem}

The first example problem was performed to show how this system could be used to speed up the process of finding the true optimum match between cycle, aircraft, and mission. Three cases were run. The first case was a baseline. The second case was run with the engine design parameters fixed and the aircraft design parameters allowed to vary. The third case was run with all the design parameters allowed to vary. For each of these cases the nozzle suppression level was assumed to be $15 \mathrm{~dB}$.

The results from these three cases can be seen in Figures 15, which shows the values of takeoff gross weight for each case.

For the first case the engine design variables were frozen at values that the engineer working on the TBE initially thought would yield an optimum solution. The nozzle suppression level was assumed to be $15 \mathrm{~dB}$. This case was run with only a takeoff field constraint being considered. There was no noise constraint. The optimizer was allowed to vary the aircraft design variables of engine and wing size. This case was used as a baseline. The takeoff field length constraint was met but the noise was well above allowable. This case was used to compare with the next two cases to determine the magnitude 
of the weight penalty necessary to meet the noise constraint.

The second case was run with the noise constraint. The engine design variables were still not allowed to vary. The only way to solve the noise problem was to oversize the engine. This case represents the initial solution to the problem using the engine originally thought to be the best solution. Both the takeoff field length and noise constraints were met. However, to meet the noise constraint the engine had to be oversized by 39 per cent at takeoff. This resulted in a significant TOGW penalty of 11 per cent as seen in Figure 15.

The third case was run with the engine design parameters, OPR and T4 Maximum, allowed to vary. Once again both the takeoff field length and noise constraints were met. However, since the engine parameters were allowed to vary, the noise constraint was met through a combination of lowering T4 Maximum and oversizing the engine. OPR stayed close to the maximum allowable value of 17.5 to maintain higher propulsive efficiency. The result was a smaller engine and a better match between engine, airframe and mission yielding a TOGW penalty of only 5.1 per cent as seen in Figure 15. This is a weight savings of over 5 per cent compared to the second case.

This is the solution an engineer would eventually achieve using the traditional approach. However, many iterations at different leveis of OPR and T4 Maximum would be required. Using the IPAS system it is possible to obtain the solution in one optimized run. This results in a time savings of approximately 80 per cent.

\section{Second Example Problem}

The second example problem was a variation of the first. For this problem it was decided to determine the affect of nozzle suppression level on the overall results. This was done by changing the amount of nozzle suppression and determining a new optimized solution. Trying to determine this effect the traditional way would require studying many different engines to determine the engine design that is optimum for each suppression level. Using the new system it is only necessary to change the suppression level in the input deck. The value of OPR was fixed at its maximum allowable value for the sake of simplicity.
Three cases were run for this example; assuming nozzle noise suppression levels of $10 \mathrm{~dB}, 15 \mathrm{~dB}$, and $20 \mathrm{~dB}$.

The results for these cases can be seen in Figures 16. Both the takeoff field length and noise constraints were met for all the cases. Figure 16 shows the aircraft weight as a function of nozzle suppression. As the amount of assumed nozzle suppression decreases the TOGW increases. This is because as the nozzle suppression goes down other tradeoffs, such as oversizing the engine and lowering the maximum turbine inlet temperature, must be made to meet the noise requirement. These methods decrease the noise but they also increase the weight.

This is an excellent example of how this system could be used for sensitivity studies. Suppose you were unsure of the level of suppression that could be obtained. Figure 16 shows how important nozzle suppression is to the overall problem. If takeoff gross weight is a strong function of the suppression level, as in this case, it is very important to invest time and money into maximizing the nozzle suppression.

Using the old method of iteration it would take approximately three weeks to obtain these results. Using the new method it would only take an estimated three optimized runs, about three days.

A major concern involved in this type of problem is how much CPU time is required to get a solution. The case with all five parameters allowed to vary required about 5 hours of CPU time on an IBM RS6000 550 workstation while the case with only two parameters allowed to varied required about 1.5 hours of CPU time. With the improvenents being made in computer technology, this time is likely to decrease rapidly.

\section{Conclusion}

The Integrated Propulsion/Airframe Analysis System shows great promise in the analysis of advanced airbreathing aerospace systems. When coupled with an optimizer, it allows solutions to be obtained in 1/5 the time previously required.

There are, however, limitations to keep in mind. In order to run a case like this it must be possible to set the problem up so that all the codes can run non interactively. This could be difficult to do. If it is 
not possible to get a reliable NEPP control scheme set up or if it is not possible to devise a scheme to determine engine weights then the traditional interactive approach must be used

\section{Future Work}

There are two areas of work needed to make this type of system more useful. First, some way must be developed to generate the engine weight as the design variables change. For the TBE it was adequate to scale the weight as a function of the design airflow. This will not be the case for other cycles.

Second, a better optimization scheme is needed. Currently, the optimizer will often get lost in the design space. Even when a constrained solution is reached, it is often not the optimum solution. If this system is to be used to its potential then the optimization scheme must give consistent results.

Finally, it is expected that the computational speed of this system can be greatly increased through the use of parallel processing.

\section{References}

1. Plencner, Robert M.; and Snyder, Christopher A.: "The Navy / Nasa Engine Program (NEPP) - A User's Manual." NASA TM-105186, 1991.

2. McCullers, L. A.: "Aircraft Configuration Optimization Including Optimized Flight Profiles, Multidisciplinary Analysis and Optimization Part I." NASA CP-2327, 1984.

3. Onat, E.; and Klees, G. W.: "A Method to Estimate Weight and Dimensions of Large and Small Gas Turbine Engines." NASA CR-159481, 1979.

4. Kowalski, Edward J.; and Atkins, Jr., Robert A.: "A Computer Code for Estimating Installed Performance of Aircraft Gas Turbine Engines, Volume I, II, III." NASA CR-159691, 159692, 159693, 1979.

5. Clark, B. J.: "A Computer Program to Predict Aircraft Noise Levels." NASA TP-1913, 1981.
6. Gesielhert, Karl A., Caddy, Michael J., and Morris, Jr., Shelby J.: "A Coniputer Program Program for Estimating Performance of Airbreathing Aircraft Engines." NASA TM-4254, 1991.

7. Seidel, Jonathan A.; Haller, William J.: and Berton, Jeffrey J.: "Comparison of Turbine Bypass and Mixed Flow Turbofan Engines for a High-Speed Civil Transport" 


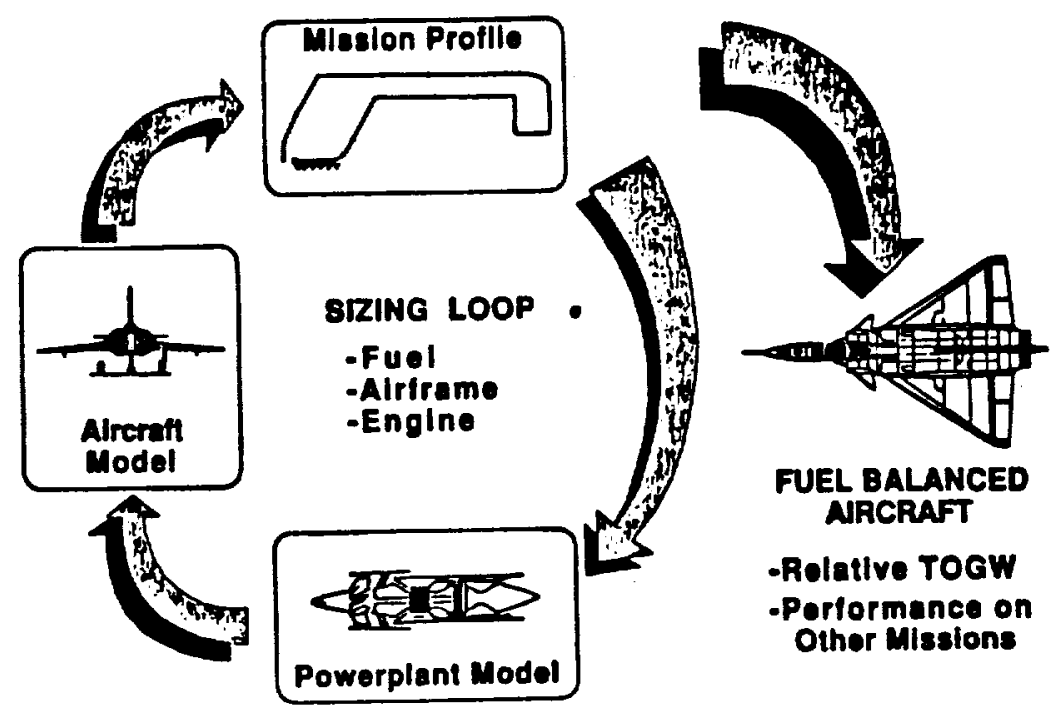

Figure 1. Aircraft Analysis Procedure

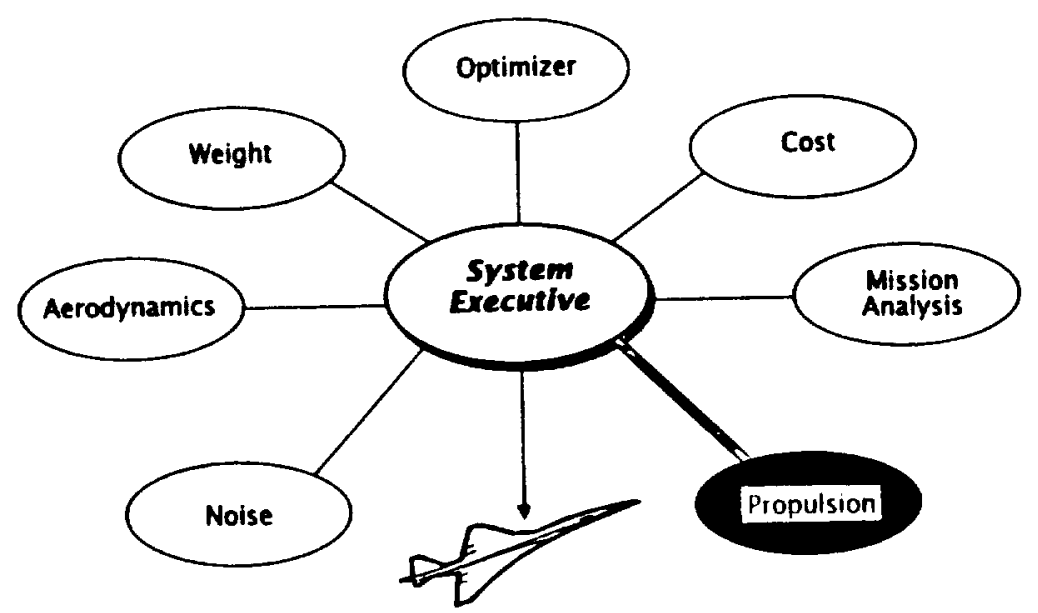

Figure 2. Aircraft Analysis System

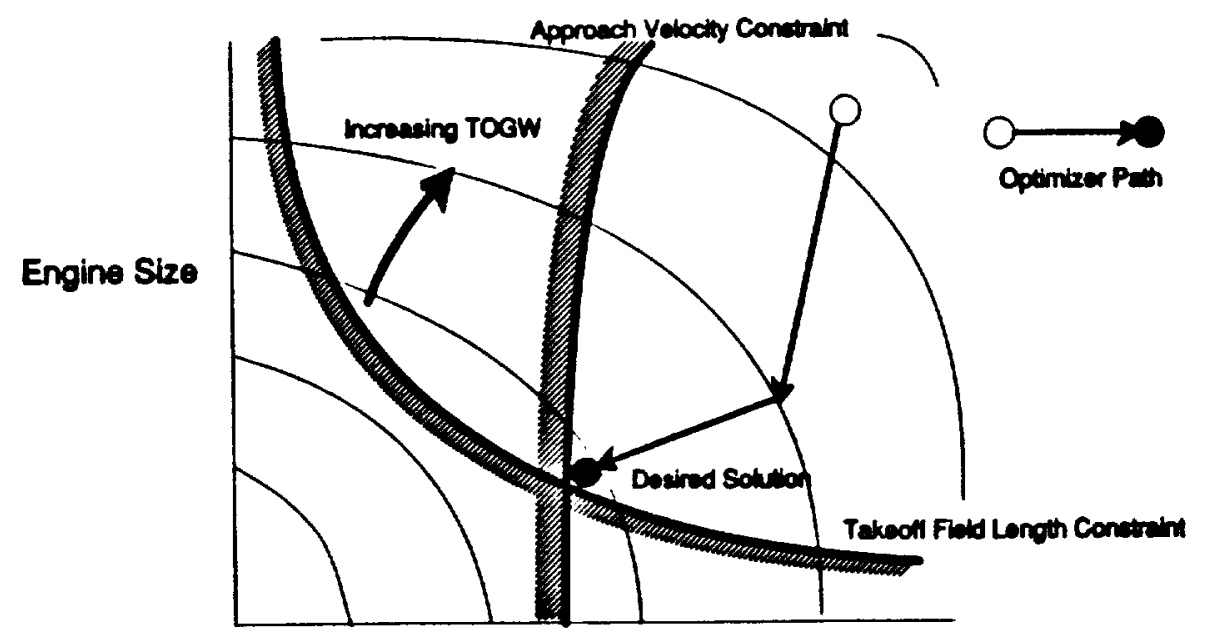

Wing Size

Figure 3. Aircraft Sizing Thumbprint 


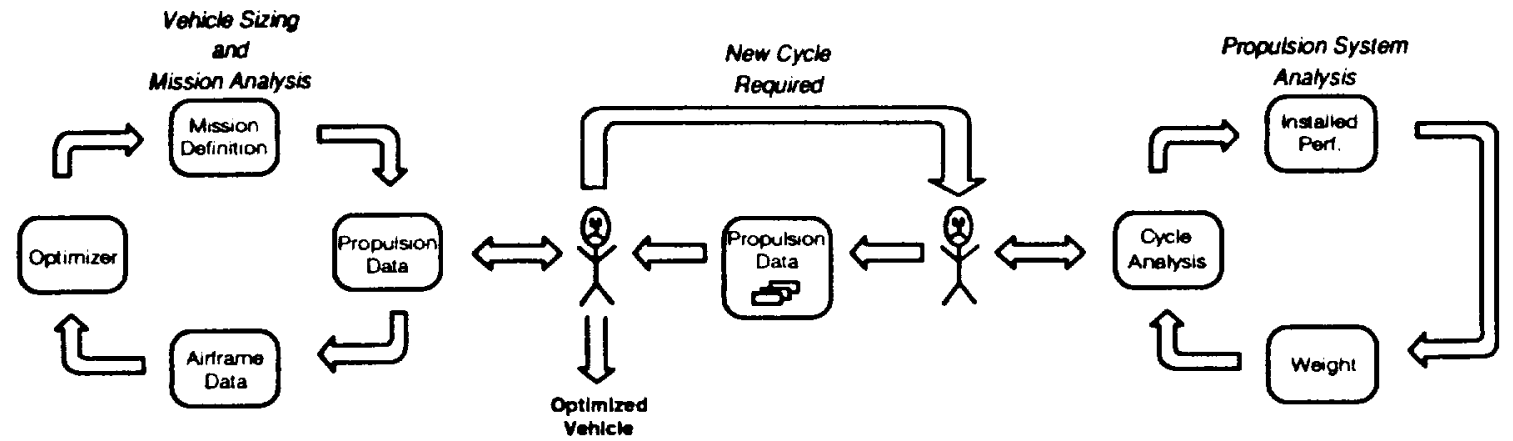

Figure 4. Propulsion System Assessment Traditional Approach

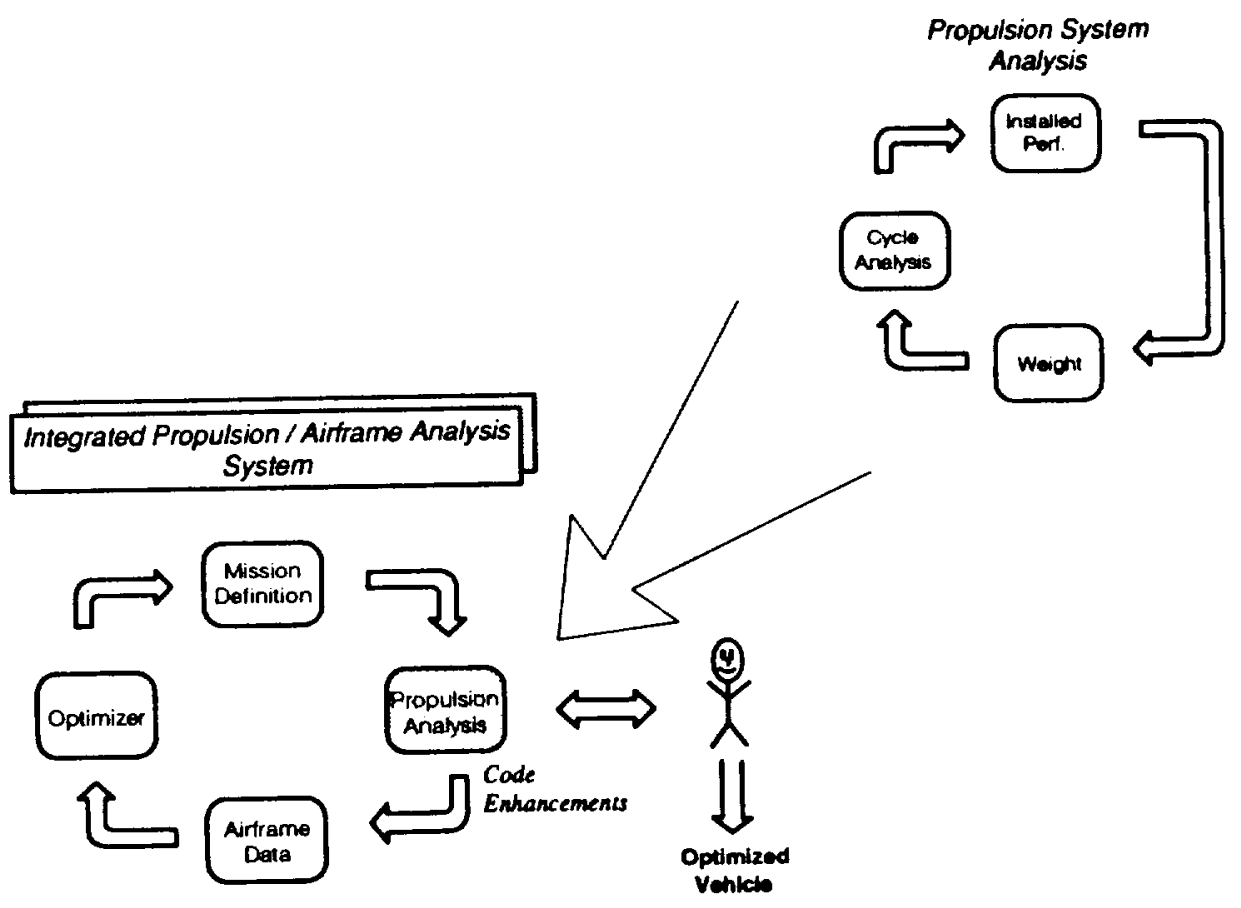

Figure 5. Propulsion System Assessment IPAS 


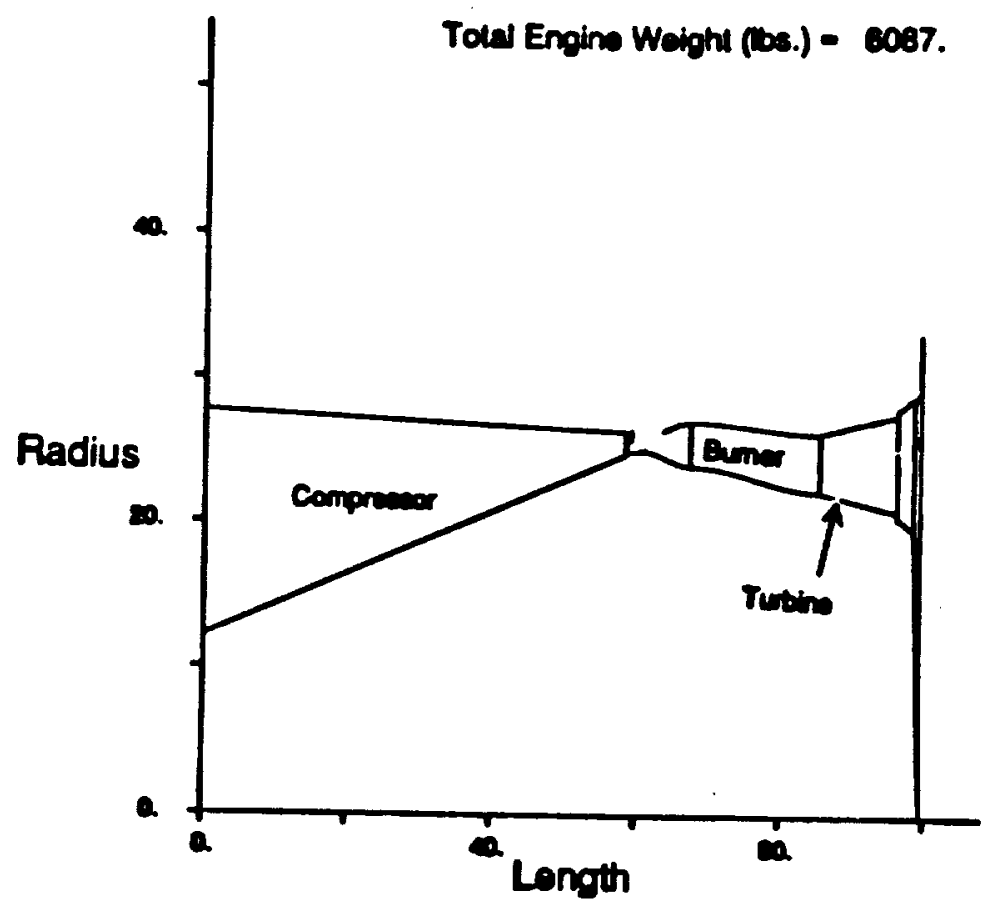

Figure 6. Acceptable TBE Flowpath

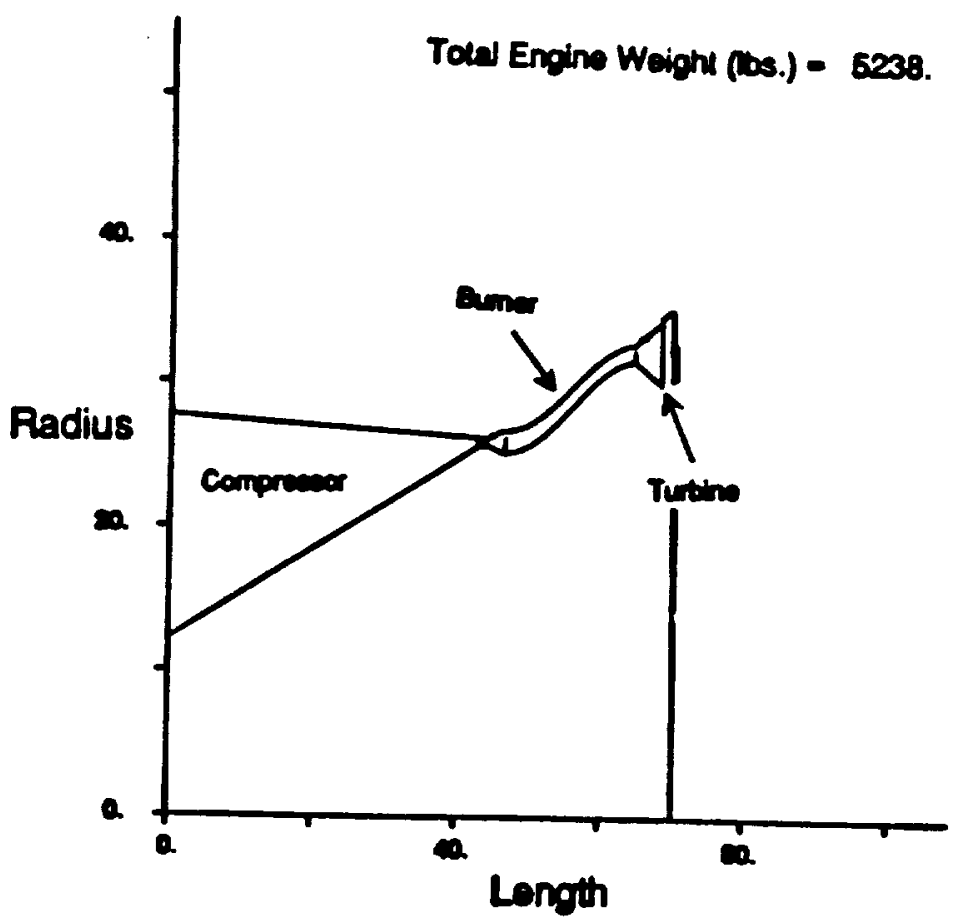

Figure 7. Unacceptable TBE Flowpath 


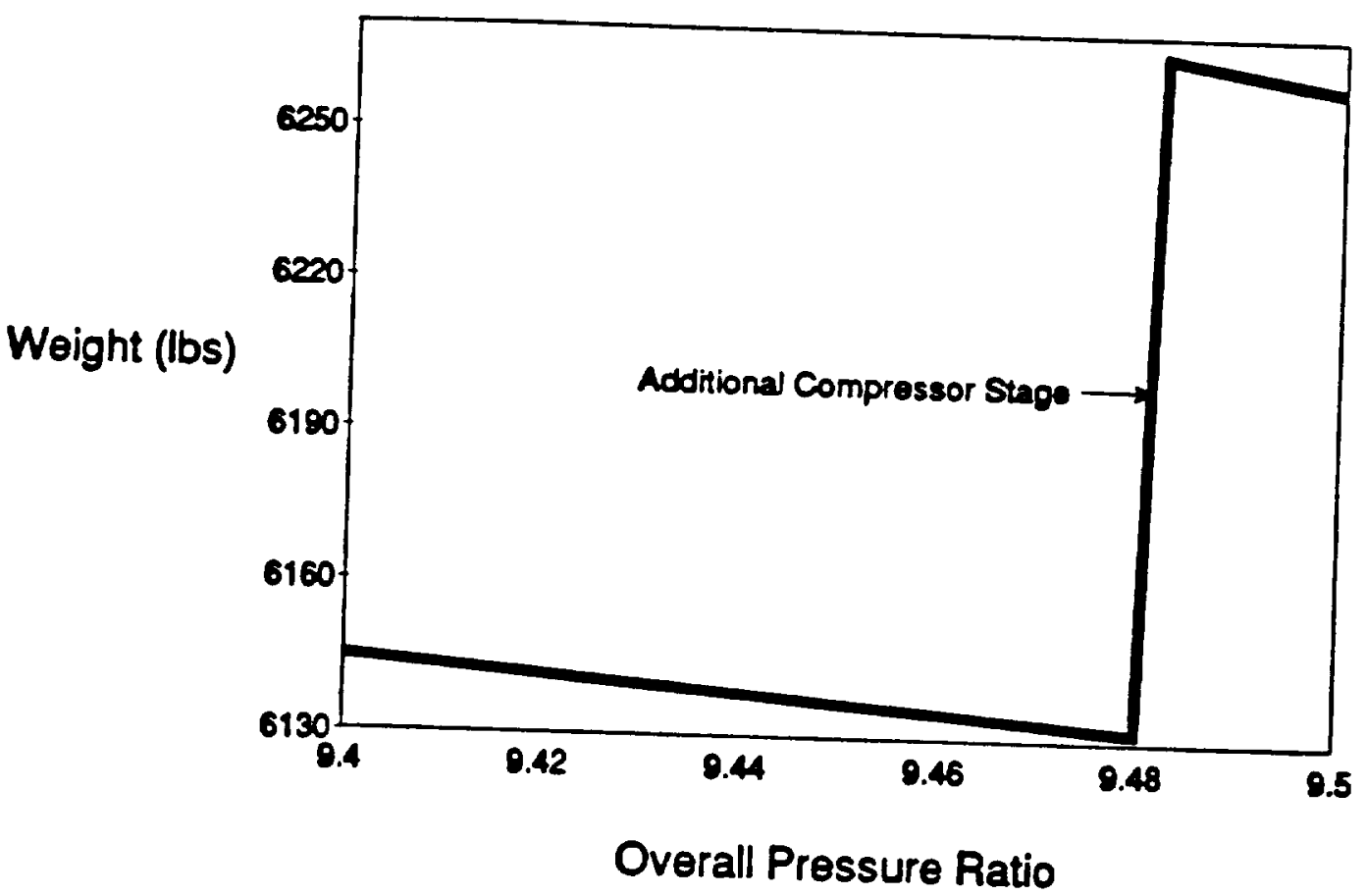

Figure 8. Step Increment in Engine Weight

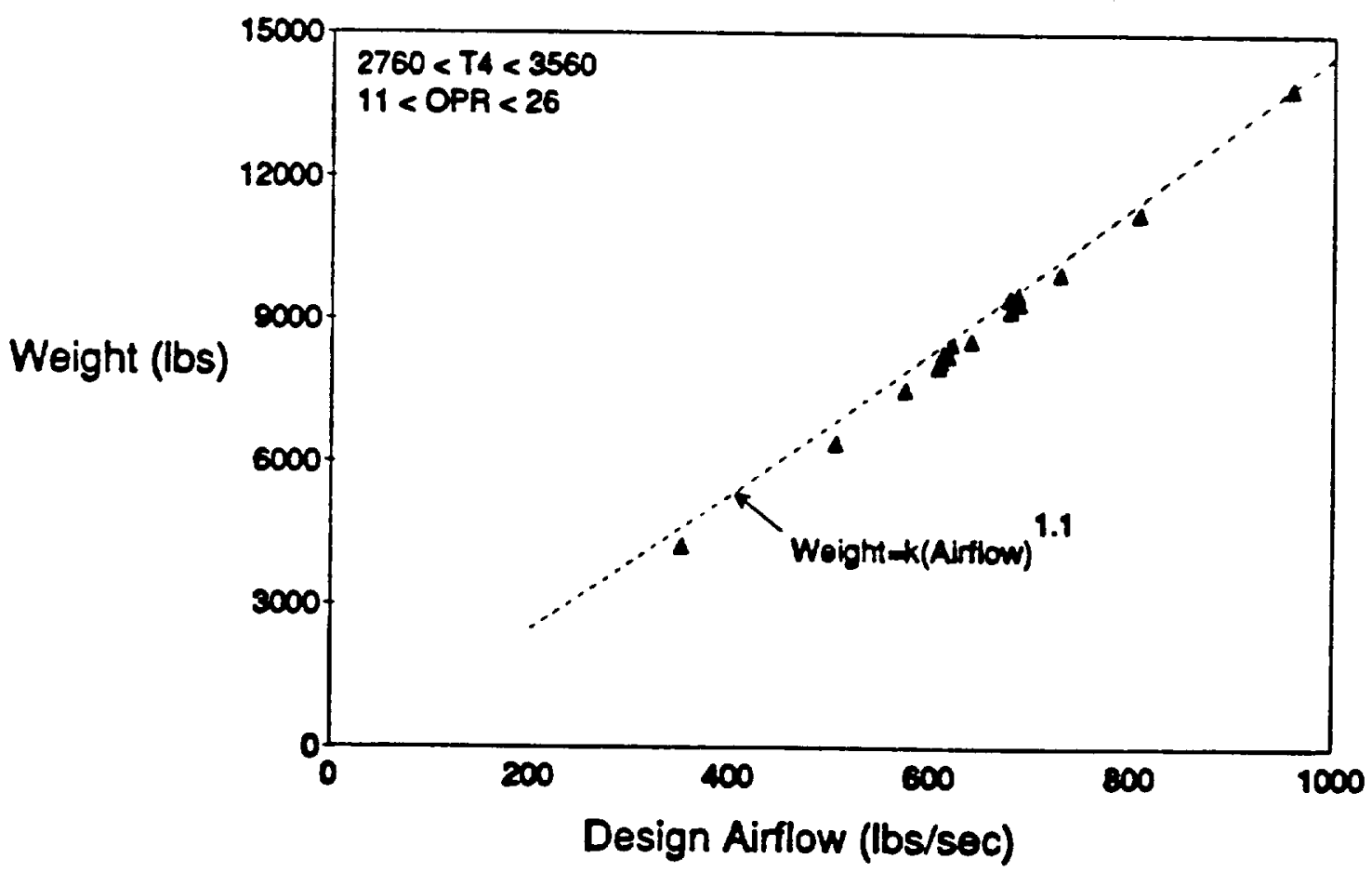

Figure 9. TBE Engine Weight Method Used 


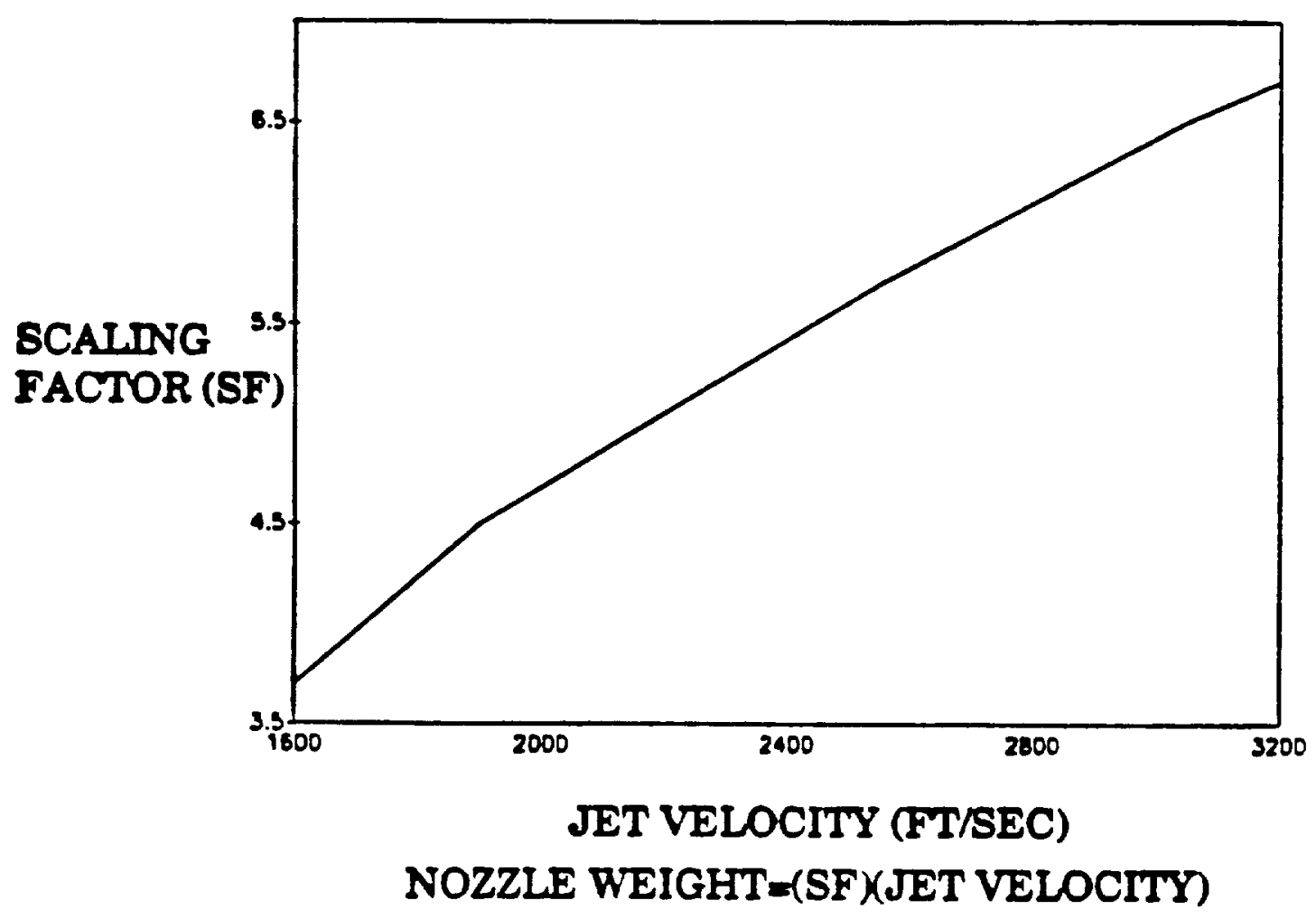

Figure 10. Mixer/Ejector Nozzle Weight

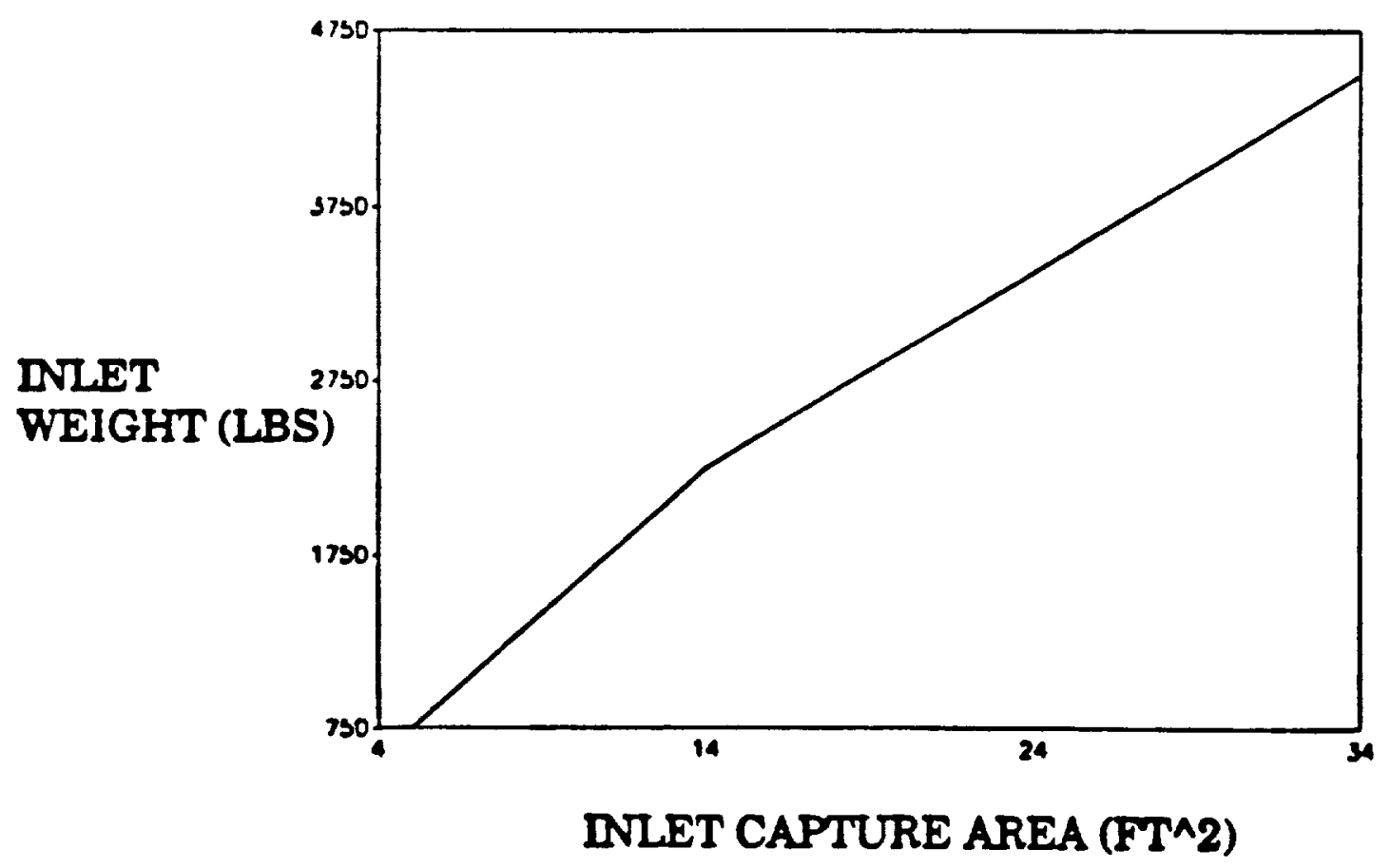

Figure 11. Inlet Weight 


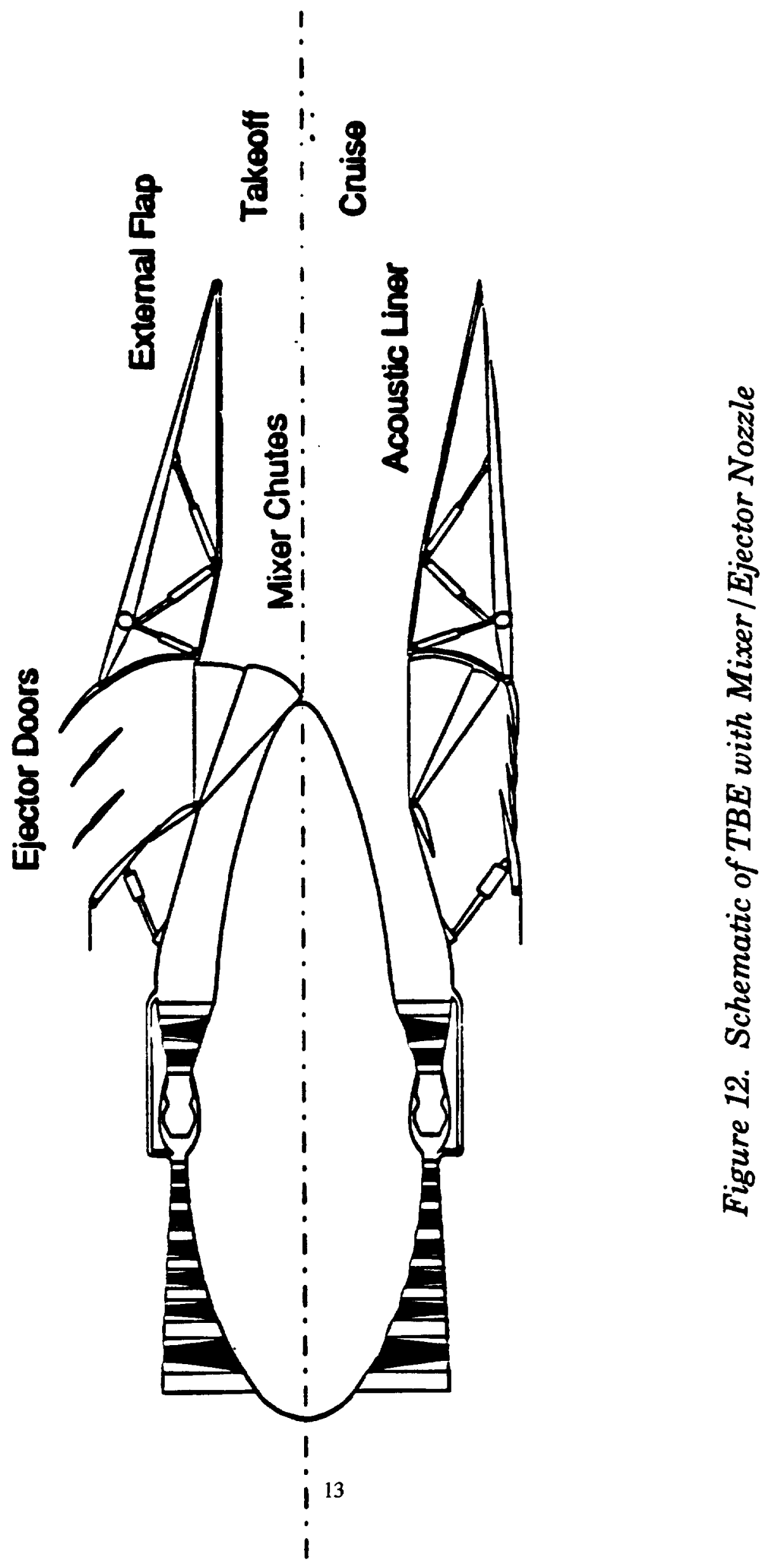




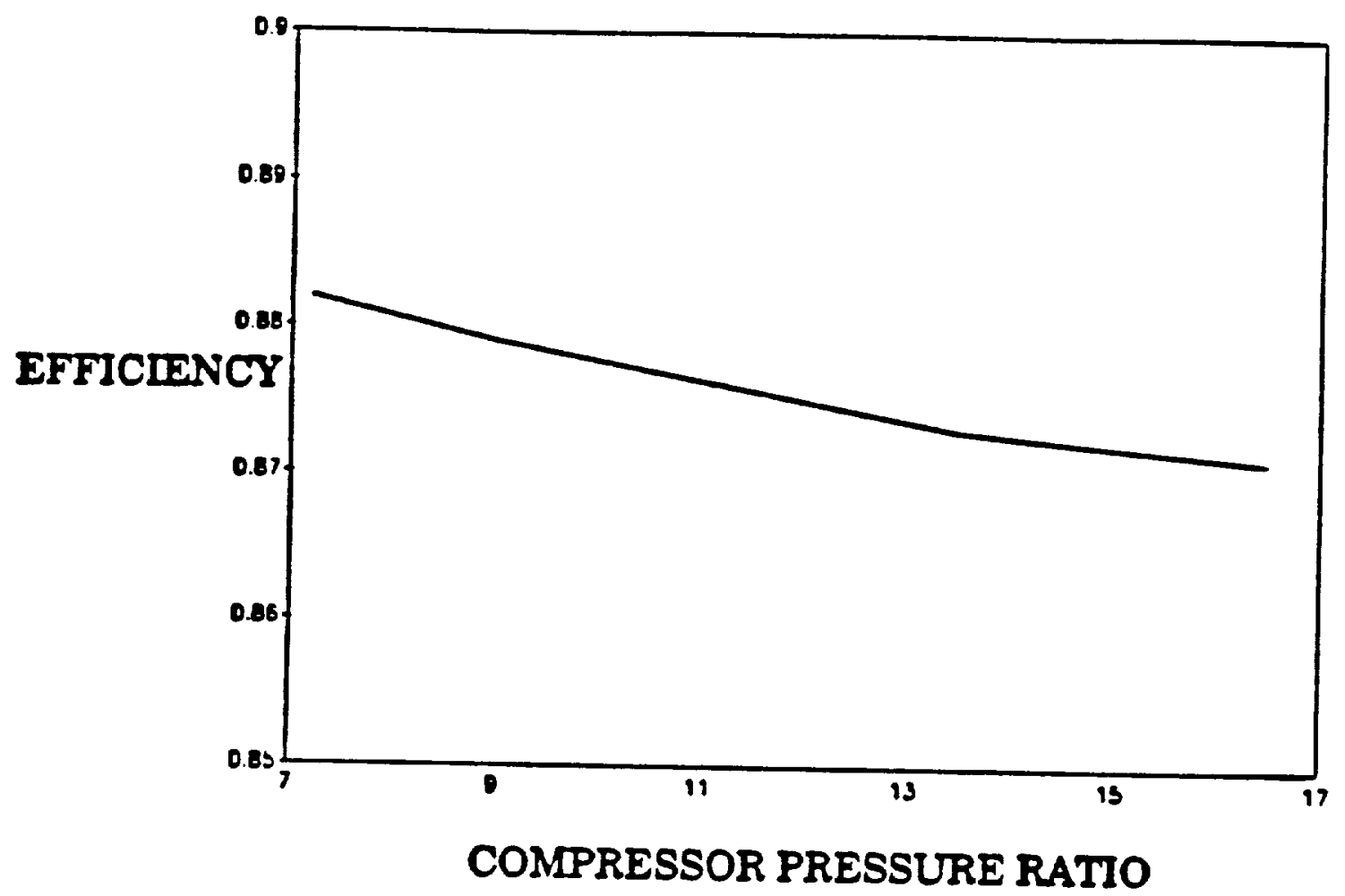

Figure 13. Compressor Efficiency Schedule

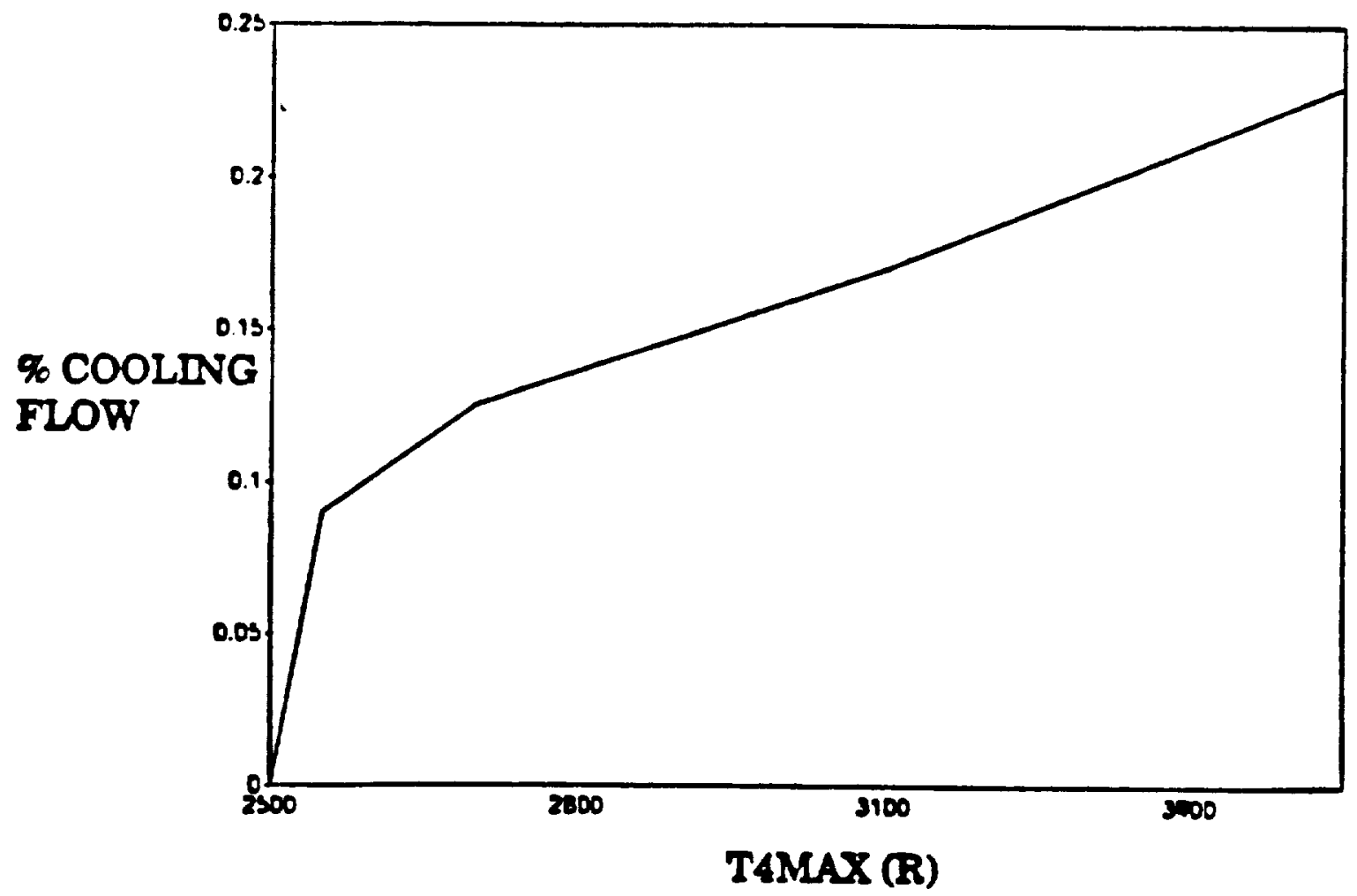

Figure 14. Turbine Cooling Flow Schedule 


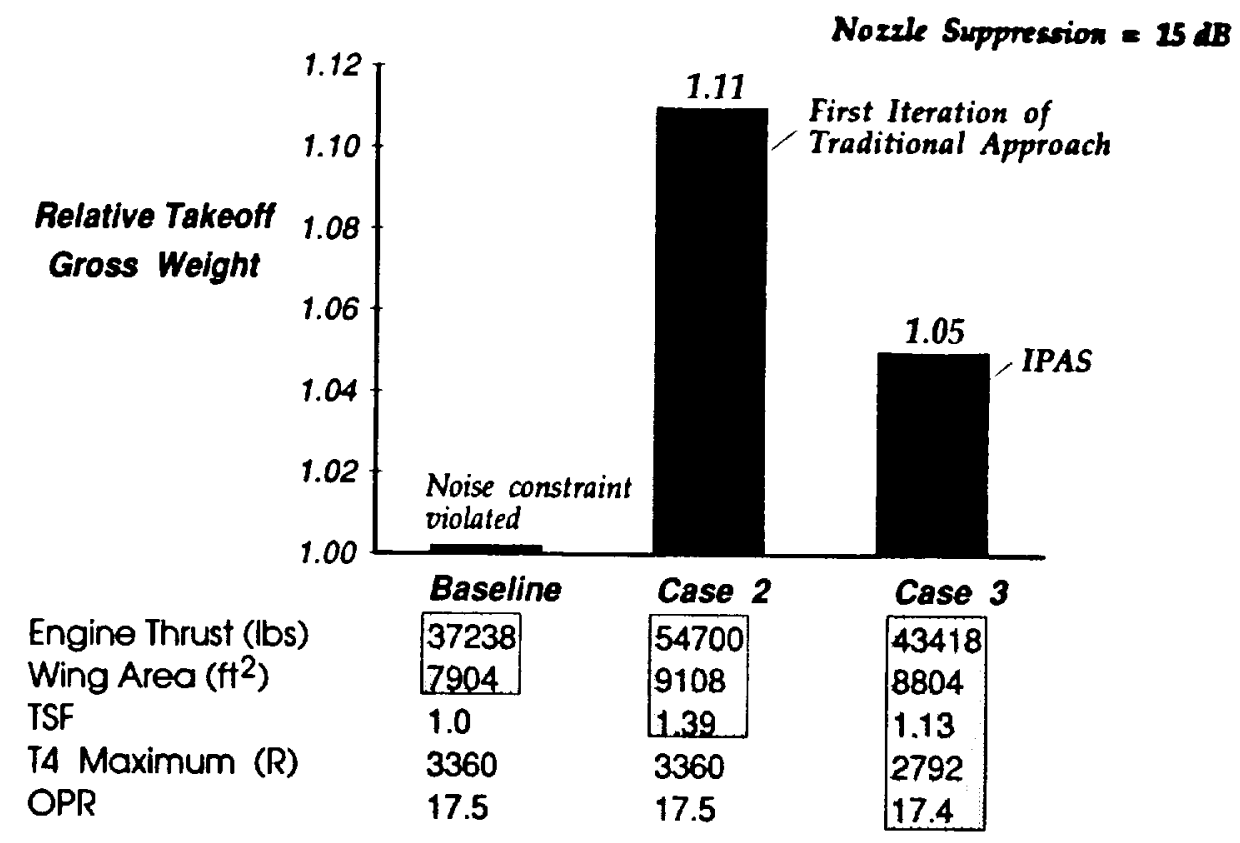

Figure 15. Comparison of Traditional and Concurrent Methods

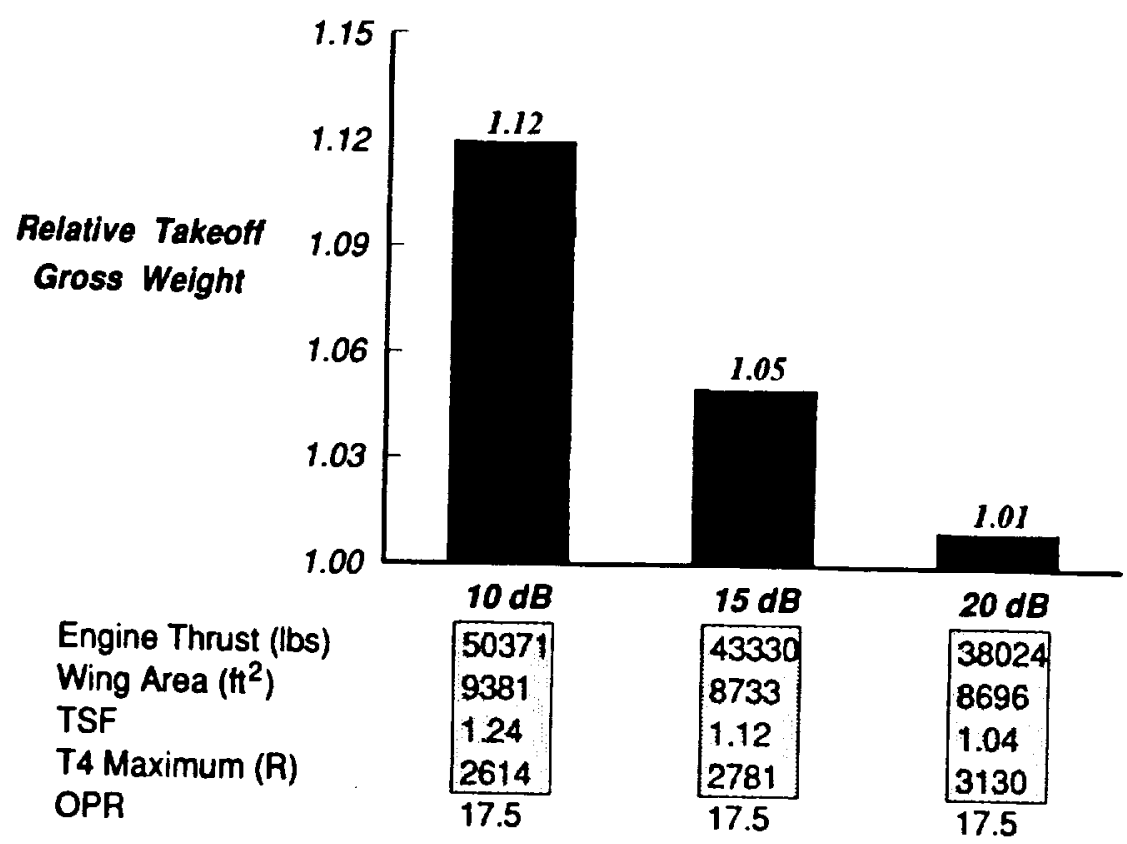

Figure 16. TOGW vs Nozzle Suppression 


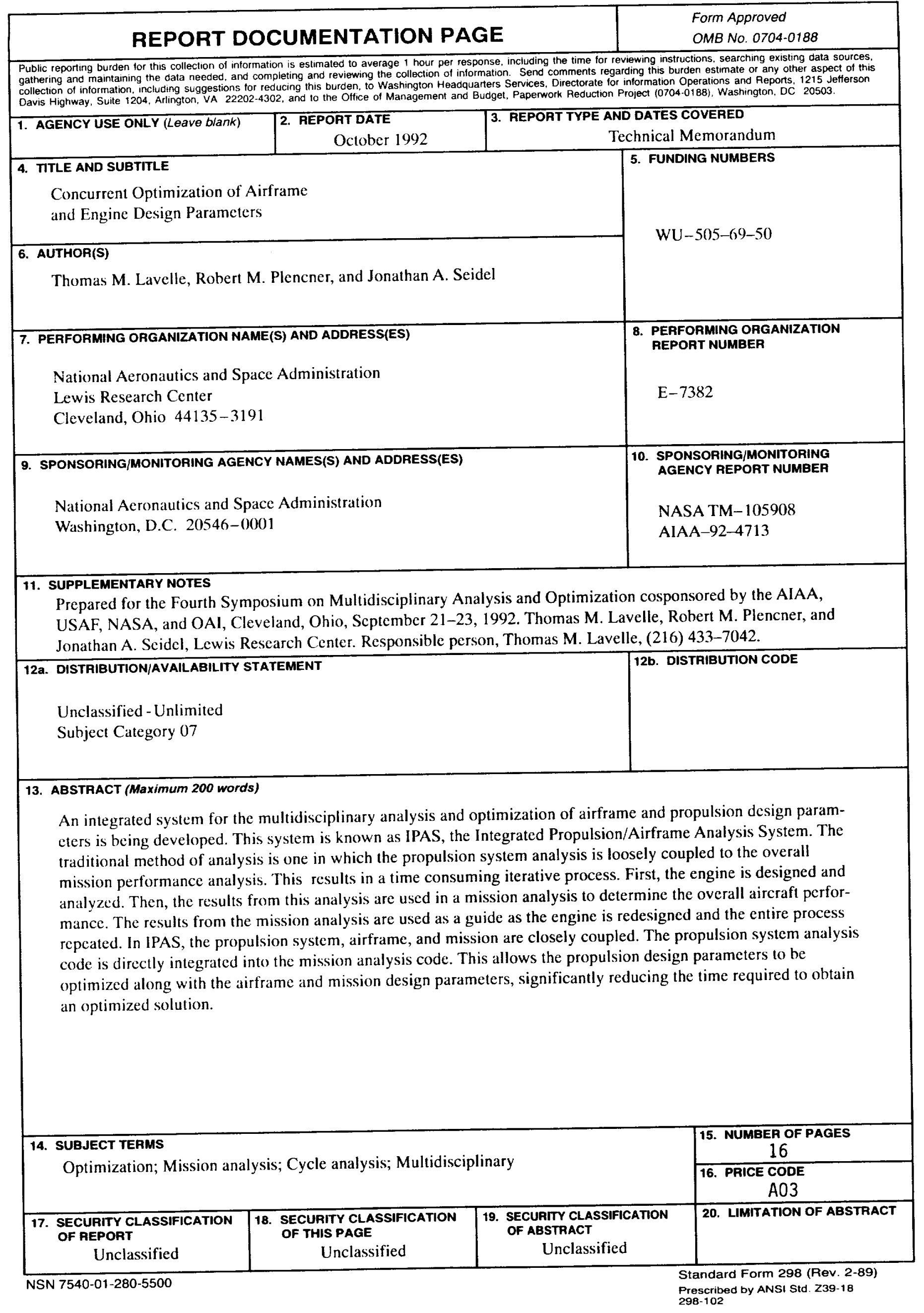

